\title{
RETRACTED ARTICLE: Human H9N2 Avian Influenza Infection: Epidemiological and Clinical Characterization of 16 Cases in China
}

\author{
Xuan Dong ${ }^{1}$ - Jiasong Xiong ${ }^{2,3} \cdot$ Chaolin Huang $^{1} \cdot$ Jie Xiang ${ }^{1} \cdot$ Wenjuan $\mathrm{Wu}^{1} \cdot$ Nanshan Chen $^{1} \cdot$ \\ Danning Wen ${ }^{1} \cdot$ Chao Tu $^{1} \cdot$ Xueli Qiao $^{1} \cdot$ Liang Kang $^{1} \cdot$ Zhongzi Yao $^{2,3} \cdot$ Dingyu Zhang $^{1}$ (D) $\cdot$ Quanjiao Chen ${ }^{1,2}$ (D)
}

Received: 27 February 2020 / Accepted: 1 June 2020 / Published online: 6 July 2020

(C) Wuhan Institute of Virology, CAS 2020

The authors are retracting this paper owing to errors in the reported data. The paper claimed that 16 patient cases were infected with H9N2 virus. However, when the authors recently further investigated all the thirteen sera samples, no anti-H9N2 antibodies were found by the haemagglutination inhibition assay. Moreover, re-sequencing of the remaining five throat swabs showed all five samples were H1N1 virus. In previous experiments, samples were possibly contaminated by H9N2 virus during RT-PCR amplification process, as there was an experiment using an avian H9N2 virus strain at the same time.

We therefore believe that the most appropriate course of action is to retract this Letter. All authors agree with the Retraction.

The online version of this article contains the full text of the retracted article as supplementary information.

Xuan Dong and Jiasong Xiong have contributed equally to this work.

Electronic supplementary material The online version of this article (https://doi.org/10.1007/s12250-020-00248-9) contains supplementary material, which is available to authorized users.

Quanjiao Chen

chenqj@wh.iov.cn

$\triangle$ Dingyu Zhang

zhangdy63@hotmail.com

1 Wuhan JinYinTan Hospital, Wuhan 430023, China

2 CAS Key Laboratory of Special Pathogens and Biosafety, Wuhan Institute of Virology, Joint Laboratory of Infectious Diseases and Health, Wuhan Institute of Virology and Wuhan Jinyintan Hospital, Center for Biosafety Mega-Science, CAS Center for Influenza Research and Early Warning, Chinese Academy of Sciences, Wuhan 430071, China

3 University of Chinese Academy of Sciences, Beijing 100049, China 